\title{
FTIR microspectroscopy of stained cells and tissues. Application in cancer diagnosis
}

\author{
Jacek K. Pijanka ${ }^{\mathrm{a}}$, Nicholas Stone ${ }^{\mathrm{b}}$, Gianfelice Cinque ${ }^{\mathrm{c}}$, Ying Yang ${ }^{\mathrm{a}}$, Achim Kohler ${ }^{\mathrm{d}, \mathrm{e}}$, \\ Katia Wehbe ${ }^{\mathrm{c}}$, Mark Frogley ${ }^{\mathrm{c}}$, Gary Parkes ${ }^{\mathrm{f}}$, Joanne Parkes ${ }^{\mathrm{f}}$, Paul Dumas ${ }^{\mathrm{g}}$, \\ Christophe Sandt ${ }^{\mathrm{g}}$, Daniel G. van Pittius ${ }^{\mathrm{f}}$, Gillian Douce ${ }^{\mathrm{f}}$, Ganesh D. Sockalingum ${ }^{\mathrm{h}}$ and \\ Josep Sulé-Suso a,i,* \\ a Institute for Science and Technology in Medicine, Guy Hilton Research Centre, Keele University, \\ Stoke on Trent, UK \\ ${ }^{\mathrm{b}}$ Biophotonics Research Unit, Leadon House, Gloucestershire Royal Hospital, Gloucester, UK \\ ${ }^{\mathrm{c}}$ Diamond Light Source, Diamond House, Harwell Science and Innovation Campus, Didcot, \\ Oxfordshire, UK \\ ${ }^{\mathrm{d}}$ Centre for Biospectroscopy and Data Modeling, Nofima-Matt Food, Ås, Osloveien, Norway \\ ${ }^{\mathrm{e}}$ CIGENE, Centre for Integrative Genetics, IMT, University of Life Sciences, Ås, Norway \\ ${ }^{\mathrm{f}}$ Department of Histopathology, Central Pathology Laboratory, University Hospital of North \\ Staffordshire, Stoke on Trent, UK \\ ' SOLEIL Synchrotron, BP48, L'Orme des Merisiers, Gif sur Yvette, France \\ ${ }^{\mathrm{h}}$ MéDIAN-Université de Reims Champagne-Ardenne, CNRS UMR6237-MEDyC, UFR de Pharmacie, \\ IFR 53, Reims, France \\ ${ }^{\mathrm{i}}$ Cancer Centre, University Hospital of North Staffordshire, Stoke on Trent, UK
}

\begin{abstract}
It is widely accepted that FTIR spectroscopy has a huge potential in cancer diagnosis. However further work is required to bring this technique into pathology departments. One of the areas where big efforts will be required is the development of cell spectra databases to be used in the diagnosis of cancer. Presently, unstained cytology and tissue samples are studied with FTIR spectroscopy. However, it is not always possible to identify in unstained samples the types of cells present. In order to achieve this, samples need staining after FTIR spectra have been obtained. We have recently shown it is possible to obtain FTIR spectra of stained cells using a synchrotron source (10.1038/labinvest.2010.8). This allows recording FTIR spectra from cells already characterized by pathologists. In order to further this work, we have now obtained FTIR spectra from stained (Papanicolau or Haematoxylin \& Eosin) single cells using a benchtop spectrometer. This would be the logical step towards a clinical application in cancer diagnosis. The data here presented show that staining caused a decreased intensity of the peaks at $2920 \mathrm{~cm}^{-1}$ and $2850 \mathrm{~cm}^{-1}$, and the appearance of stronger peaks at $1374 \mathrm{~cm}^{-1}$ and $1040 \mathrm{~cm}^{-1}$. The clinical applications are discussed.

Keywords: FTIR spectroscopy, benchtop spectrometer, synchrotron, cancer, Papanicolau, Haematoxylin \& Eosin
\end{abstract}

\footnotetext{
${ }^{*}$ Corresponding author: Dr Josep Sulé-Suso, Institute for Science and Technology in Medicine, Guy Hilton Research Centre, Keele University, Thornburrow Drive, Stoke on Trent ST4 7QB, UK. Tel.: +44 1782 672571; Fax: +44 1782 672601; E-mail: josep.sulesuso@uhns.nhs.uk.
} 


\section{Introduction}

Over the last few years, it has been shown that FTIR spectroscopy could become a powerful tool in the diagnosis of cancer. While the clinical application is not here yet, the increasing number of publications on the subject confirms this belief (reviewed by Andrus) [1]. One of the main steps towards a clinical application will be the development of cell spectra databases for diagnostic purposes. This, however, will involve a huge workload and time. At present, FTIR spectra are usually obtained from unstained cytology and/or tissue samples. This entails the difficulty of identifying, before staining, the types of cells present in the sample. However, this could be made faster if FTIR spectra could be obtained from already stained samples. This would allow first, pathologists to identify the cells of interest and, second, spectroscopists concentrating on obtaining FTIR spectra of those cells deemed important for the setting up of such databases.

To our knowledge, the only example of FTIR spectra recorded from stained samples was carried out by Dukor [2] using breast tissue sections mounted on a glass slide and studied with FTIR attenuated total reflectance (ATR) microspectroscopy. However, it is more difficult to obtain FTIR spectra of a single, chosen cell using ATR microspectroscopy when compared to FTIR microspectroscopy using transmission or reflection modalities. We have previously shown that it is possible to obtain FTIR spectra of already stained single cells on MirrIR low-e microscope slides using a synchrotron source [9]. However, pathological diagnosis of cancer entails, amongst other, studying cytology and/or tissue samples. Furthermore, should this technique prove to be useful in cancer diagnosis, benchtop instruments will need to be available at Pathology Departments rather than using synchrotrons, which in spite of their important biomedical applications [3] could lengthen the whole diagnostic process. Therefore, the aim of this study was to assess whether FTIR spectra could be obtained, using a benchtop spectrometer, not only from stained cells seeded on MirrIR low-e microscope slides but also from single cells present in already stained tissues.

\section{Materials and methods}

\subsection{Cell lines}

The lung epithelial cell line NL20 and the epidermoid lung cancer cell line CALU-1, purchased from the American Type Culture Collection (ATCC, LGC Standards, Teddington, UK) and the European Collection of Cell Cultures (ECACC, Salisbury, UK), respectively, were used in this study. NL 20 cells were kept in culture in Ham's F12 (Lonza, UK) supplemented with 4\% Foetal Calf Serum, insulin, epidermal growth factor, transferrin and hydrocortisone according to the provider's instructions. CALU1 cells were kept in culture in DMEM with $10 \%$ Foetal Calf Serum. Cells were incubated at $37^{\circ} \mathrm{C}$ in $5 \%$ $\mathrm{CO}_{2}$ in tissue culture flasks (Sarstedt, UK).

\subsection{Cell growth on MirrIR low-e microscope slides}

Cell growth on MirrIR low-e microscope slides (Kevley Technologies, Chesterland, OH, USA) has been previously described [8]. Briefly, cells growing in tissue culture flasks were detached before reaching confluence with trypsin/EDTA (Sigma, UK) and collected by centrifugation at $1200 \mathrm{rpm}$ for $7 \mathrm{~min}$. Supernatant was removed, and the cell pellet was resuspended in $5 \mathrm{ml}$ of culture media. Cell viability was assessed using the trypan blue exclusion method. Cells were then seeded at $5 \times 10^{4}$ cells in $200 \mu \mathrm{l}$ 
of complete media on MirrIR low-e microscope slides and incubated for $2 \mathrm{~h}$ at $37^{\circ} \mathrm{C}$ and $5 \% \mathrm{CO}_{2}$ to allow them to attach. Following this, slides were incubated in a Petri dish with $15 \mathrm{ml}$ of complete media for $22 \mathrm{~h}$ at $37^{\circ} \mathrm{C}$ and $5 \% \mathrm{CO}_{2}$.

In order to confirm that exactly the same cells were studied with FTIR spectroscopy pre- and poststaining, a grid was drawn on MirrIR low-e microscope slides using a diamond pen as previously described [9]. This approach allowed identification of each individual cell based on row and column numbers.

\subsection{Cell and tissue staining}

Lung cancer and lung epithelial cell seeding on MirrIR low-e microscope slides and fixation have been previously described [9]. Cell samples were stained with Papanicolaou (Pap) using the same standard staining protocol used at the Pathology Department at the University Hospital of North Staffordshire (UHNS). Lung tissue samples were obtained following ethical approval from already stored tissue in paraffin. $5 \mu \mathrm{m}$ thickness sections were cut, dewaxed and stained with Hematoxylin \& Eosin (H\&E) at the Pathology Department, UHNS. H\&E includes a first step staining with haematoxylin and then eosin while Pap staining includes staining with haematoxylin, Orange G and EA50 in this order. More important, H\&E staining includes one washing with ethanol after eosin staining but not after haematoxylin. However, Pap staining includes ethanol washings after haematoxylin, Orange G and EA50 stains are added to the sample.

\subsection{FTIR microspectroscopy}

FTIR spectra of single cells on MirrIR low-e microscope slides and in lung tissue were obtained using a Spotlight 400 benchtop spectrometer (Perkin-Elmer) available at the laboratory facilities at Gloucestershire Royal Hospital, and a Bruker spectrometer (vertex 80v) available at Diamond Facility (in offline mode). Both instruments have a microscope fitted with both white light and infrared optics and a liquid nitrogen cooled MCT detector. Spectra were collected at $4 \mathrm{~cm}^{-1}$ resolution using a double path single masking aperture size of $20 \times 20 \mu \mathrm{m}^{2}$ in order to include whole single cells. Spectra were processed and corrected for the Mie scatter using Extended Multiplicative Signal Correction [6].

Also, spectra of single cells within lung tissue were recorded at the SMIS beamline of the Soleil Synchrotron facilities (Saint-Aubin, France). A Nicolet Nexus FTIR spectrometer coupled to a Nicolet Continuum XL IR microscope fitted with $32 \times$ infinity corrected Schwarzschild objective and equipped with liquid nitrogen cooled MCT/A detector. Spectra were collected at $4 \mathrm{~cm}^{-1}$ resolution using a double path single masking aperture size of $15 \times 15 \mu \mathrm{m}^{2}$.

\section{Results}

\subsection{FTIR spectra of single stained cells using a benchtop spectrometer}

FTIR spectra of exactly the same CALU-1 and NL20 cells pre- and post-Pap staining were obtained using a benchtop spectrometer. Figure 1 shows the mean of 50 spectra of 50 individual CALU- 1 and 50 individual NL20 cells pre- and post-staining. The main differences were seen in the lipids area with a decreased intensity of the peaks at $2920 \mathrm{~cm}^{-1}$ and $2850 \mathrm{~cm}^{-1}$ following staining with Pap. Other changes were the appearance of stronger peaks at $1374 \mathrm{~cm}^{-1}$ and $1040 \mathrm{~cm}^{-1}$ following staining with Pap. 


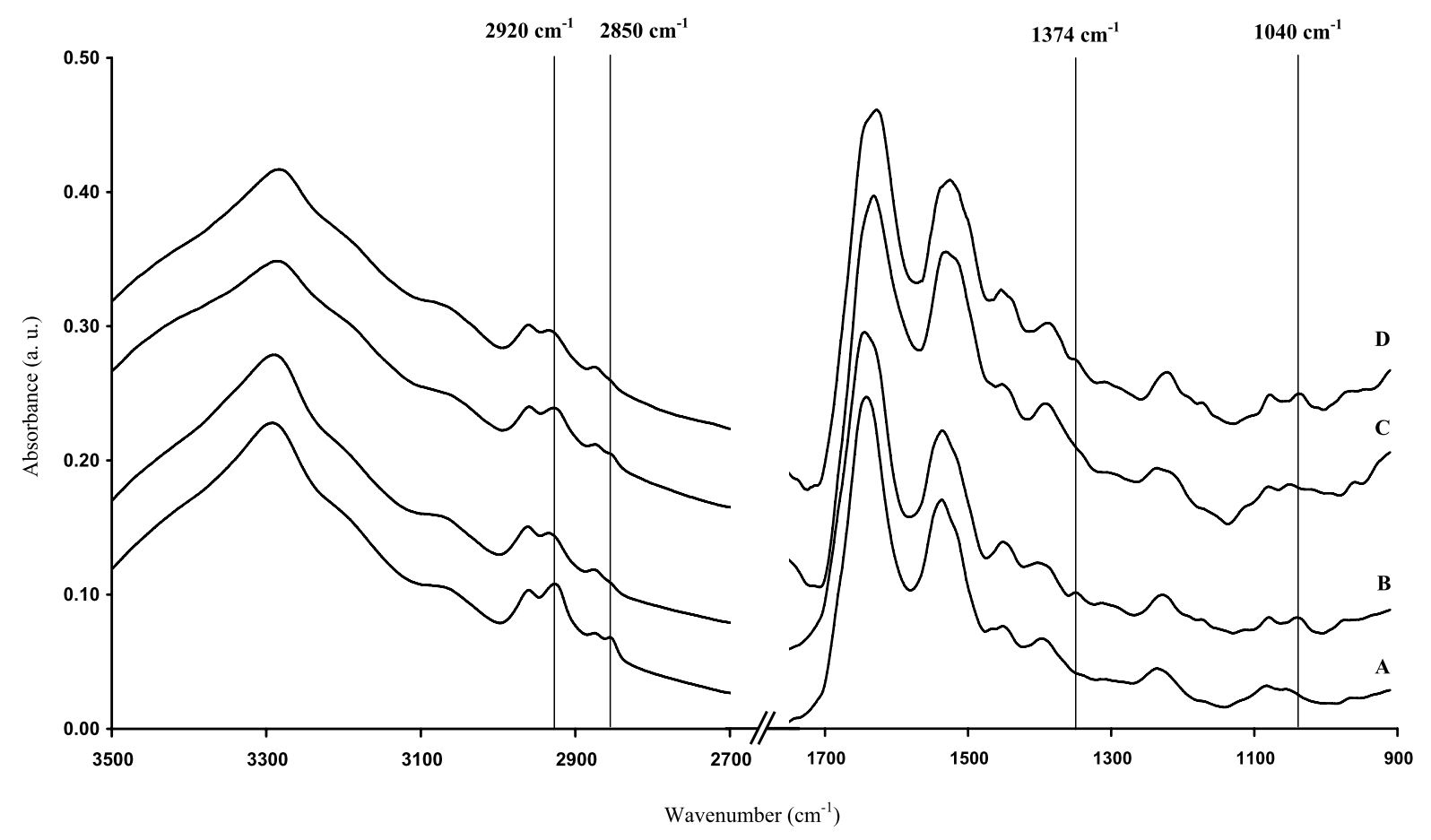

Fig. 1. Mean spectrum of 50 FTIR spectra from the same 50 individual CALU-1 cells prior (A) and post (B) Pap staining, and from the same individual NL20 cells prior (C) and post (D) Pap staining. Spectra are offset for clarity.

\subsection{FTIR spectra of single cells in stained lung tissue}

We studied whether FTIR spectra could be obtained from single cells within H\&E stained tissue using either synchrotron or a benchtop spectrometer. The advantage of analyzing stained tissue is that the infrared beam can be more easily focused directly onto one single cell decreasing spectral contamination from surrounding tissue and/or cells. Figure 2 shows it is possible to obtain FTIR spectra with good signal to noise ratios of a single lung cancer cell using either a synchrotron source or a benchtop spectrometer. Again the same changes in the lipids area (a decrease of the intensity of the peaks at $2920 \mathrm{~cm}^{-1}$ and $2850 \mathrm{~cm}^{-1}$ ) were seen as described in the spectra of single lung cancer and lung epithelial cells following staining with Pap. These confirm that the changes in the lipids are due to the staining procedure. On the other hand, a peak was also seen at $1374 \mathrm{~cm}^{-1}$ in the spectra of stained cancer cells studied with either a synchrotron source or a benchtop spectrometer (Fig. 2) suggesting this could be another spectral change caused by the staining procedure.

\section{Discussion}

$\mathrm{H} \& \mathrm{E}$ and Pap staining techniques are widely used in pathology departments. H\&E is mainly used to stain tissue samples while Pap is used to stain cytology samples. The advantage of using stained samples in FTIR spectroscopy is that individual cells can be identified and classified (normal, dysplasia, cancer, reactive, and so on) by a pathologist/cytologist before obtaining their FTIR spectra [9]. Therefore, it is important to confirm that FTIR spectra with good signal to noise ratio can be obtained from single cells 


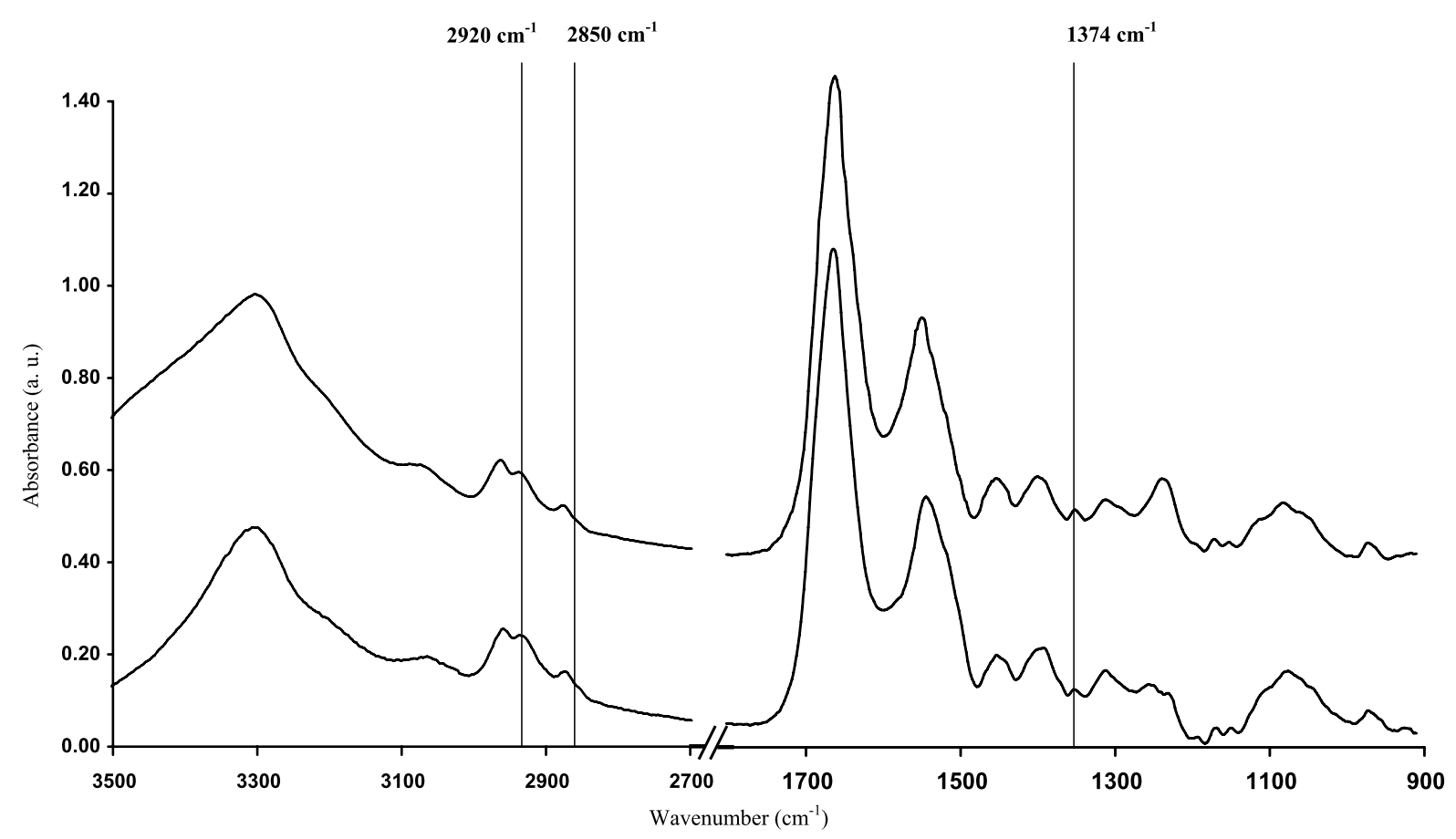

Fig. 2. Representative FTIR spectra of 2 lung cancer cells in an already H\&E stained lung tissue sample obtained with a synchrotron source (bottom spectrum) and a benchtop spectrometer (top spectrum). Spectra are offset for clarity.

in already stained tissues if a clinical application in cancer diagnosis is to be achieved. Furthermore, for this technique to be widely used in a hospital set up, it is important to confirm that good quality spectra can be obtained from such samples using a benchtop spectrometer.

The study of Pap stained single cells on MirrIR low-e microscope slides using a benchtop spectrometer confirmed the changes seen in the lipid area using synchrotron based FTIR spectroscopy [9]. These are the disappearance of the peaks at $2920 \mathrm{~cm}^{-1}$ and $2850 \mathrm{~cm}^{-1}$ corresponding to $\mathrm{CH}_{2}$ stretching modes of methylene chains in membrane lipids $[7,4,11]$ caused by ethanol washings during the Pap staining process (Fig. 1). This is further confirmed by the fact that the same changes in the lipid area were seen when obtaining the FTIR spectra of single cells in H\&E stained tissue samples using either a benchtop spectrometer or synchrotron (Fig. 2). On the other hand, we saw a new peak at $1374 \mathrm{~cm}^{-1}$ in both stained cytology and tissue samples. Paraffin has an absorption peak at around $1378 \mathrm{~cm}^{-1}$ [10]. It could be argued that this peak in tissue samples could still be due to remains of paraffin. However, the fact that this peak also appeared in cytology samples devoid of paraffin (Fig. 1) would indicate that the emergence of this peak is due to the staining process. Furthermore, haematoxylin (a component of both H\&E and Pap which gives the strongest peaks) has a broad band at $1350-1450 \mathrm{~cm}^{-1}$ [9]. The peak at $1040 \mathrm{~cm}^{-1}$ was seen in cells on MirrIR low-e microscope slides stained with Pap (Fig. 1) but not in cells present in tissue samples and stained with H\&E. This peak has been associated with the carbohydrate signal $\nu_{\mathrm{s}}(\mathrm{C}-\mathrm{O}-\mathrm{C})$ [5]. It is not possible at this stage to say whether the appearance of this peak following staining with Pap is due to the staining process itself as haematoxylin has a strong peak at $1050 \mathrm{~cm}^{-1}$ but not at $1040 \mathrm{~cm}^{-1}$ [9]. We are carrying out further work to better understand these changes.

The data showed in this feasibility study confirms that it is possible to obtain FTIR spectra of both stained cells and tissues using a benchtop spectrometer. This opens up a huge array of possibilities in 
the pathological diagnosis of cancer as it will allow obtaining FTIR spectra of already pathologically characterized cells. Some of the benefits of this procedure will be the increased speed in setting up spectral databases of cells as spectroscopists could now focus directly on the cells of interest. Also, the study of already stained cells with FTIR spectroscopy will allow pathologists to further characterise those cells deemed abnormal but not necessarily diagnostic of cancer. The latter could lead towards a quicker diagnosis of cancer rather than waiting for a further biopsy with all the risks, side effects and costs that this entails for patients and the NHS.

\section{Acknowledgements}

We acknowledge SOLEIL synchrotron for provision of synchrotron radiation facilities at beamline SMIS (proposal number 20080402). Diamond Light Source is also acknowledged for the use off-line of B22 beamline facility (IR end station 1).

\section{References}

[1] P.G. Andrus, Cancer monitoring by FTIR spectroscopy, Technol. Cancer Res. Treat. 5 (2006), 157-167.

[2] R.K. Dukor, M.N. Liebman and B.L. Johnson, A new non-destructive method for analysis of clinical samples with FT-IR microspectroscopy. Breast cancer tissue as an example, Cell. Mol. Biol. 44 (1998), 211-217.

[3] P. Dumas, G.D. Sockalingum and J. Sulé-Suso, Adding synchrotron radiation to infrared microspectroscopy: what's new in biomedical applications?, Trends Biotechnol. 25 (2007), 40-44.

[4] T. Gao, J. Feng and Y. Ci, Human breast carcinoma tissues display distinctive FTIR spectra: implications for the histological characterisation of carcinomas, Anal. Cell. Pathol. 18 (1999), 87-93.

[5] E. Gazi, J. Dwyer, N.P. Lockyer, P. Gardner, J.H. Shanks, J. Roulson, C.A. Hart, N.W. Clarke and M.D. Brown, Biomolecular profiling of metastatic prostate cancer cells in bone marrow tissue using FTIR microspectroscopy: a pilot study, Anal. Bioanal. Chem. 387 (2007), 1621-1631.

[6] A. Kohler, J. Sulé-Suso, G.D. Sockalingum, M. Tobin, F. Bahrami, Y. Yang, J. Pijanka, P. Dumas, M. Cotte, D.G. Van Pittius, G. Parkes and H. Martens, Estimating and correcting Mie scattering in synchrotron-based microscopic FTIR spectra by extended multiplicative signal correction (EMSC), Appl. Spectrosc. 62 (2008), 259-266.

[7] P. Lasch, M. Boese, A. Pacifico and M. Diem, FT-IR spectroscopy investigations of single cells on the subcellular level, Vibr. Spectrosc. 28 (2002), 147-157.

[8] J. Pijanka, A. Kohler, Y. Yang, P. Dumas, S. Chio-Srichan, M. Manfait, G.D. Sockalingum and J. Sulé-Suso, Spectroscopic signatures of single, isolated cancer cell nuclei using synchrotron infrared microscopy, Analyst 134 (2009), 1176-1181.

[9] J. Pijanka, G.D. Sockalingum, A. Kohler, Y. Yang, F. Draux, P. Dumas, C. Sandt, G. Parkes, D.G. van Pittius, G. Douce, V. Untereiner and J. Sulé-Suso, Synchrotron based FTIR spectra of stained single cells. Towards a clinical application in pathology, Lab. Invest., doi: 10.1038/labinvest.2010.8.

[10] A. Tfayli, O. Piot, A. Durlach, P. Bernard and M. Manfait, Discriminating nevus and melanoma on paraffin-embedded skin biopsies using FTIR microspectroscopy, Biochim. Biophys. Acta 1724 (2005), 262-269.

[11] J. Zhou, Z. Wang, S. Sun, M. Liu and H. Zhang, A rapid method for detecting conformational changes during differentiation and apoptosis of HL60 cell by Fourier Transform infrared spectroscopy, Biotechnol. Appl. Biochem. 33 (2001), $127-132$. 


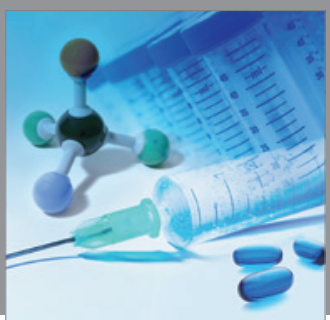

International Journal of

Medicinal Chemistry

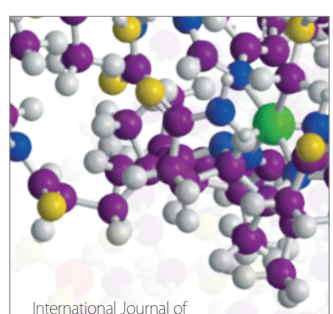

Carbohydrate Chemistry

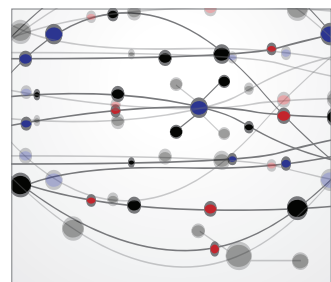

The Scientific World Journal
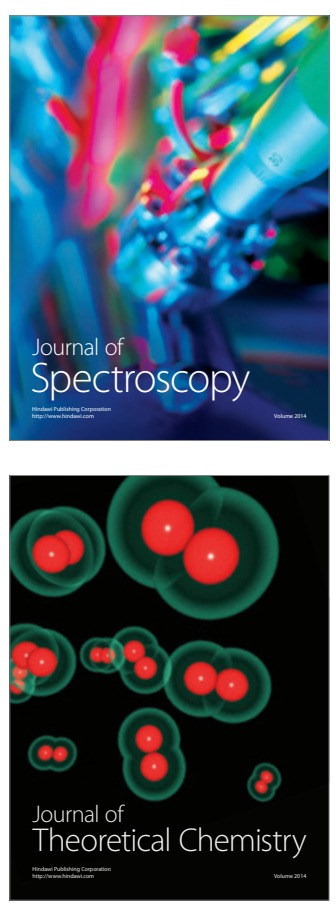
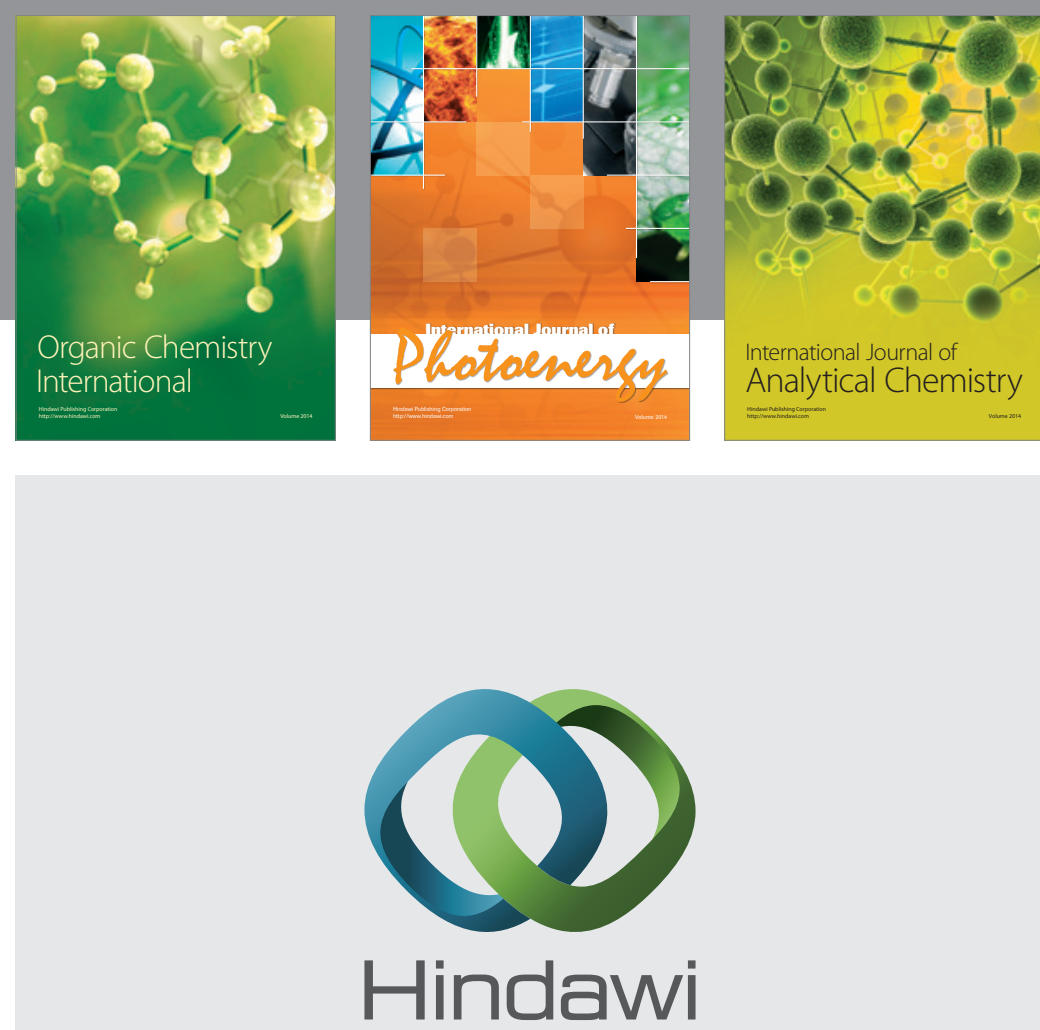

Submit your manuscripts at

http://www.hindawi.com
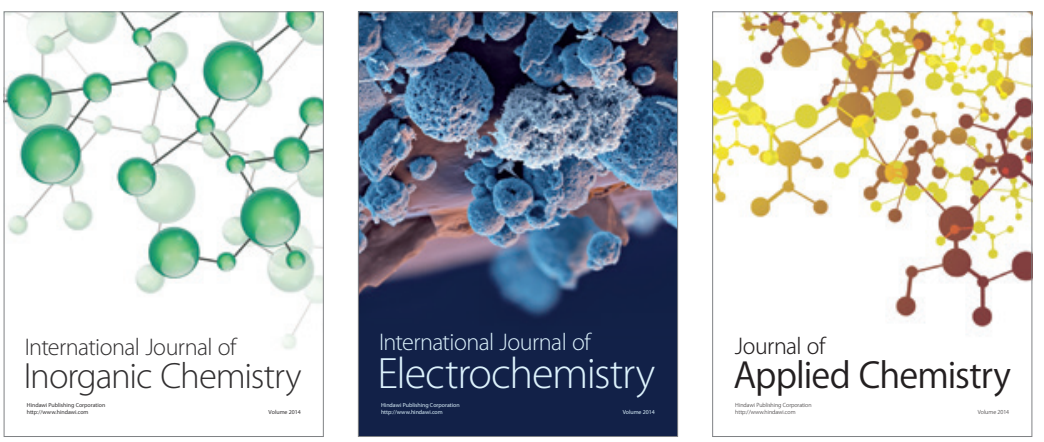

Journal of

Applied Chemistry
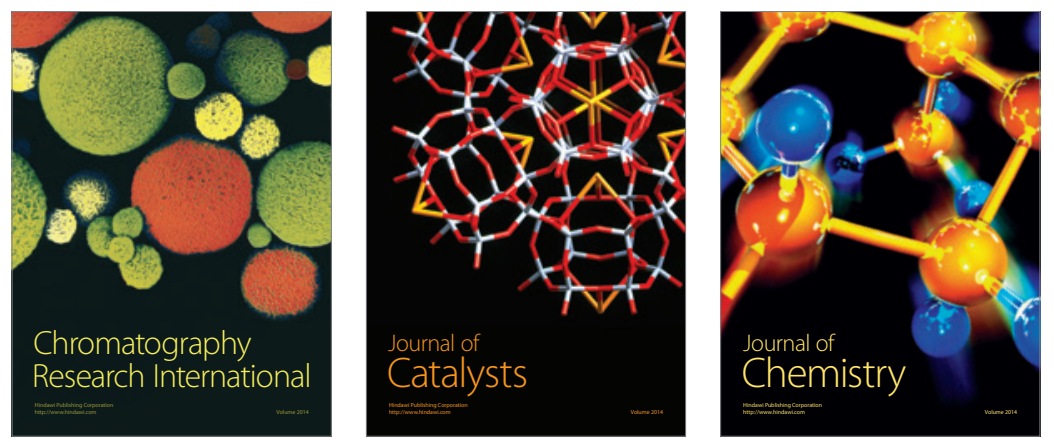
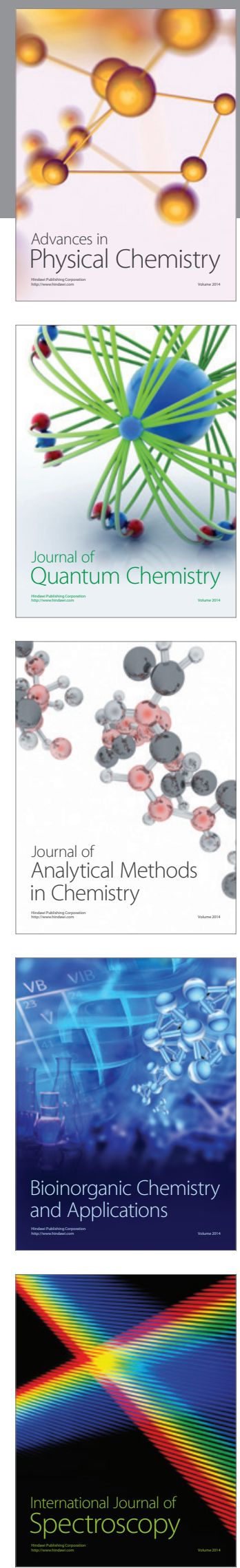